\title{
A Few Complex Equations Constituted by an Operator Consisting of Fractional Calculus and Their Consequences
}

\author{
Hüseyin Irmak ${ }^{1}$ and Basem Aref Frasin ${ }^{2}$ \\ ${ }^{1}$ Department of Mathematics, Faculty of Science, Çankırı Karatekin University, 18100 Çankırı, Turkey \\ ${ }^{2}$ Department of Mathematics, Faculty of Science, Al Al-Bayt University, P.O. Box 130095, Mafraq 25113, Jordan
}

Correspondence should be addressed to Hüseyin Irmak; hisimya@yahoo.com

Received 18 September 2013; Accepted 10 November 2013; Published 29 January 2014

Academic Editors: Y. Feng and Z.-Y. Li

Copyright (c) 2014 H. Irmak and B. A. Frasin. This is an open access article distributed under the Creative Commons Attribution License, which permits unrestricted use, distribution, and reproduction in any medium, provided the original work is properly cited.

A few complex (differential) equations constituted by certain operators consisting of fractional calculus are first presented and some of their comprehensive consequences relating to (analytic and) geometric function theory are then pointed out.

\section{Introduction, Definitions, Notations, and Motivation}

From the literature, as we know, fractional calculus (FC) is a generalization of ordinary differentiation and integration to arbitrary noninteger order. This subject is as old as the differential calculus and also goes back to time when Leibniz and Newton invented differential calculus. The efficient usage of FC has been a subject of interest not only among mathematicians but also among physicists and engineers, appearing in rheology, viscoelasticity, electrochemistry, electromagnetism, and so forth. For example, see the works in the references in [1-11].

Fractional differential equations (FDE), that is, differential equations determined by FC, have also many applications in modeling of physical and chemical processes and in engineering. In their turn, mathematical aspects of studies on FDC were discussed by several authors. For those, can be also seen the works in [3, 12-29].

As we have emphasized just above, the main purpose of this investigation is both to present a novel work relating to analytic and/or geometric function theory (AGFT) and FDC and to reveal some (comprehensive) results between certain complex valued functions and complex (differential) equations constituted by certain operators dealing with FC. In particular, special consequences of the main results are also pointed out in the concluding section of this paper.

Now, there is a need to introduce some notations and definitions which will be used in this work.

First, let $\mathbb{R}, \mathbb{C}$, and $\mathbb{N}$ be the set of real numbers, the set of complex numbers and the set of positive integers, respectively. Also let $\mathbb{N}_{0}:=\mathbb{N} \cup\{0\}, \mathbb{C}^{*}:=\mathbb{C}-\{0\}$, and $\mathbb{R}^{*}:=\mathbb{R}-\{0\}$.

For $0 \leq \mu<1$ and an analytic function $\kappa:=\kappa(z)$, the symbol $\mathscr{D}_{z}^{\mu}[\kappa]$ denotes an operator of FC, which is defined as follows (cf., e.g., $[9,30-33])$.

Let $\kappa(z)$ be an analytic function in a simply-connected region of the $z$-plane containing the origin. Then, the fractional derivative of order $\mu$ is defined by

$$
\mathscr{D}_{z}^{\mu}[\kappa]=\frac{1}{\Gamma(1-\mu)} \frac{d}{d z} \int_{0}^{z} \frac{\kappa(\xi)}{(z-\xi)^{\mu}} d \xi \quad(0 \leq \mu<1),
$$

where the multiplicity of $(z-\xi)^{-\mu}$ above is removed by requiring $\log (z-\xi)$ to be real when $z-\xi>0$. Note that, here and throughout this investigation, the function $\Gamma$ is the wellknown gamma function.

Under the hypotheses of the definition above, for an analytic function $\kappa(z)$, the fractional derivative of order $m+\mu$ is also defined by

$$
\mathscr{D}_{z}^{m+\mu}[\kappa]=\frac{d^{m}}{d z^{m}}\left(\mathscr{D}_{z}^{\mu}[\kappa]\right) \quad\left(0 \leq \mu<1 ; m \in \mathbb{N}_{0}\right) .
$$


By means of (1) and (2), for a function $\kappa(z)=z^{\nu}$, it can be easily determined that

$$
\mathscr{D}_{z}^{m+\mu}\left[z^{\nu}\right]=\frac{\Gamma(\nu+1)}{\Gamma(\nu-m-\mu+1)} z^{\nu-m-\mu}
$$

for some $0 \leq \mu<1$ and for all $m \in \mathbb{N}_{0}$ with $m<\nu-\mu+1$.

In the usual notation, let $\mathscr{A}$ denote the family of the functions $f(z)$ normalized by the following Taylor-Maclaurin series:

$$
\begin{array}{r}
f(z)=z+a_{2} z^{2}+a_{3} z^{3}+\cdots+a_{n} z^{n}+\cdots \\
\left(a_{n+1} \in \mathbb{C} ; n \in \mathbb{N}\right),
\end{array}
$$

which are analytic in the unit open disk $\mathbb{U}=\{z: z \in \mathbb{C}$ and $|z|<1\}$. Also let $\mathcal{S}$ denote the family of functions belonging to $\mathscr{A}$ which are univalent in the open disk $\mathbb{U}$. As is known, the functions family $\mathscr{A}$ has important roles for AGFT (see $[34,35])$. In particulare, some of the important and wellinvestigated families of the univalent function family $\mathcal{S}$ include the family $\mathcal{S}^{*}(\tau)$ of starlike functions of order $\tau$ and the family $\mathscr{K}(\tau)$ of convex functions of order $\tau(0 \leq \tau<1)$ in the domain $\mathbb{U}$. As their definitions, we also recall

$$
\begin{aligned}
& \mathcal{S}^{*}(\tau):=\left\{f \in \mathcal{S}: \mathfrak{R} e\left(\frac{z f^{\prime}(z)}{f(z)}\right)>\tau(0 \leq \tau<1 ; z \in \mathbb{U})\right\}, \\
& \mathscr{K}(\tau):=\left\{f \in \mathcal{S}: \mathfrak{R} e\left(1+\frac{z f^{\prime \prime}(z)}{f^{\prime}(z)}\right)>\tau(0 \leq \tau<1 ; z \in \mathbb{U})\right\} .
\end{aligned}
$$

It readily follows from definitions (5) that $f(z) \in \mathscr{K}(\tau)$ if and only if $z f^{\prime}(z) \in \mathcal{S}^{*}(\tau)$. For their details, one may refer to $[34,35]$.

Now, by using the operator $\mathscr{D}_{z}^{\mu}[\cdot]$, for a function $f(z)$ in $\mathscr{A}$ (or, in $\mathcal{S}$ ), given by (1), we can again define a linear operator $J_{z}^{\mu}[f]$ as in the following form:

$$
\begin{aligned}
J_{z}^{\mu}[f] & =\Gamma(2-\mu) z^{\mu} \mathscr{D}_{z}^{\mu}[f] \\
& =z+\sum_{k=2}^{\infty} \frac{\Gamma(k+1) \Gamma(2-\mu)}{\Gamma(k-\mu+1)} a_{k} z^{k},
\end{aligned}
$$

where $\mu \in \mathbf{R}:=\mathbb{R}-\{2,3,4, \ldots\}$.

\section{Main Result and Certain Consequences}

In this section, firstly, in order to prove main result, we need to recall the following assertion given by [36].

Lemma 1. Let $p(z)$ be an analytic function in the disk $\mathbb{U}$ with $p(0)=1$. If there exists a point $z_{0}$ in $\mathbb{U}$ such that

$$
\begin{aligned}
& \mathfrak{R} e(p(z))>0 \quad\left(|z|<\left|z_{0}\right|\right), \\
& \mathfrak{R} e\left(p\left(z_{0}\right)\right)=0, \quad p\left(z_{0}\right) \neq 0,
\end{aligned}
$$

then

$$
\begin{aligned}
& p\left(z_{0}\right)=i a, \\
& \left.\frac{z p^{\prime}(z)}{p(z)}\right|_{z=z_{0}}=i k\left(a+\frac{1}{a}\right) \quad\left(k \geq 1 ; a \in \mathbb{R}^{*}\right) .
\end{aligned}
$$

The comprehensive main result involving certain analytic functions and complex equations constituted by FC, which is fractional derivatives, is contained in Theorem 2 below.

Theorem 2. Let $\psi(z)$ be an analytic function and satisfy any of the following inequalities:

$$
\mathfrak{R} e(\psi(z)) \neq 0, \quad|\Im m(\psi(z))|<2
$$

and also let the function $\mathscr{F}(z)$ be defined in the following form:

$$
\mathscr{F}(z)=(1-\lambda) f(z)+\lambda z f^{\prime}(z) \quad(0 \leq \lambda \leq 1 ; f(z) \in \mathcal{S}) .
$$

$$
\begin{aligned}
& \text { If } \mathscr{F}:=\mathscr{F}(z) \text { satisfies the following complex equation: } \\
& \qquad z \cdot\left(\frac{J_{z}^{\alpha}[\mathscr{F}]}{J_{z}^{\beta}[\mathscr{F}]}\right)^{\prime}-\psi(z) \cdot \frac{J_{z}^{\alpha}[\mathscr{F}]}{J_{z}^{\beta}[\mathscr{F}]}=-\gamma \cdot \psi(z),
\end{aligned}
$$

then

$$
\mathfrak{R} e\left(\frac{J_{z}^{\alpha}[\mathscr{F}]}{J_{z}^{\beta}[\mathscr{F}]}\right)>\gamma \quad(0 \leq \gamma<1 ; \alpha \in \mathbf{R} ; \beta \in \mathbf{R} ; z \in \mathbb{U}) .
$$

Proof. For a function $f(z) \in \mathcal{S}$, we assume that $\mathscr{F}(z)$ has got the form as in (10). Then, in view of $J_{z}^{\alpha}[\mathscr{F}]$ together with (6), it can be easily derived that

$$
\begin{aligned}
\frac{J_{z}^{\alpha}[\mathscr{F}]}{J_{z}^{\beta}[\mathscr{F}]}= & \frac{\Gamma(2-\alpha) z^{\alpha} \mathscr{D}_{z}^{\alpha}[\mathscr{F}]}{\Gamma(2-\beta) z^{\beta} \mathscr{D}_{z}^{\beta}[\mathscr{F}]} \\
= & \left(\left(z+\sum_{k=2}^{\infty} \frac{(k \lambda-\lambda+1) \Gamma(k+1) \Gamma(2-\alpha)}{\Gamma(k-\alpha+1)} a_{k} z^{k}\right)\right. \\
& \left.\times\left(z+\sum_{k=2}^{\infty} \frac{(k \lambda-\lambda+1) \Gamma(k+1) \Gamma(2-\beta)}{\Gamma(k-\beta+1)} a_{k} z^{k}\right)^{-1}\right) \\
=: & h(z),
\end{aligned}
$$

where $0 \leq \gamma<1, \alpha \in \mathbf{R}, \beta \in \mathbf{R}$, and $z \in \mathbb{U}$. Clearly, the function $h(z)$ has a removable singular point at $z=0$ and also its series expansion is in the following form:

$$
h(z)=1+h_{1} z+h_{2} z^{2}+h_{3} z^{3}+\cdots \quad(z \in \mathbb{U}) .
$$

It follows from equality (13) along with (14) that one can define a function $p(z)$ by

$$
\frac{1}{1-\gamma} \cdot\left(\frac{J_{z}^{\alpha}[\mathscr{F}]}{J_{z}^{\beta}[\mathscr{F}]}-\gamma\right)=p(z) \quad(0 \leq \gamma<1 ; z \in \mathbb{U}) .
$$

Obviously, $p(z)$ has a similar series expansion to the function $h(z)$ in (14) and is an analytic function in $\mathbb{U}$ with $p(0)=1$. By the help of equality (15), we obtain

$$
\frac{z \cdot\left[(1 /(1-\gamma)) \cdot\left(J_{z}^{\alpha}[\mathscr{F}] / J_{z}^{\beta}[\mathscr{F}]-\gamma\right)\right]^{\prime}}{(1 /(1-\gamma)) \cdot\left(J_{z}^{\alpha}[\mathscr{F}] / J_{z}^{\beta}[\mathscr{F}]-\gamma\right)}=\frac{z p^{\prime}(z)}{p(z)}
$$


or, equivalently,

$$
\frac{z \cdot\left(J_{z}^{\alpha}[\mathscr{F}] / J_{z}^{\beta}[\mathscr{F}]\right)^{\prime}}{J_{z}^{\alpha}[\mathscr{F}] / J_{z}^{\beta}[\mathscr{F}]-\gamma}\left(=\frac{z p^{\prime}(z)}{p(z)}\right)=\psi(z) .
$$

Suppose now that there exists a point $z_{0}$ in $\mathbb{U}$ such that

$$
\begin{aligned}
& \mathfrak{R} e(p(z))>0 \quad\left(|z|<\left|z_{0}\right|\right), \\
& \mathfrak{R} e\left(p\left(z_{0}\right)\right)=0, \quad p\left(z_{0}\right) \neq 0 .
\end{aligned}
$$

Then, from (8) of Lemma 1, we obtain that

$$
\begin{aligned}
& p\left(z_{0}\right)=i a, \\
& \left.\frac{z p^{\prime}(z)}{p(z)}\right|_{z=z_{0}}=i k\left(a+\frac{1}{a}\right) \quad\left(k \geq 1 ; a \in \mathbb{R}^{*}\right) .
\end{aligned}
$$

If we use the related equations in (17), we then arrive at

$$
\begin{aligned}
& \mathfrak{R} e\left(\psi\left(z_{0}\right)\right)=\mathfrak{R} e\left(\left.\frac{z p^{\prime}(z)}{p(z)}\right|_{z=z_{0}}\right)=0, \\
& \left|\mathfrak{I} m\left(\psi\left(z_{0}\right)\right)\right|=\left|\mathfrak{\Im} m\left(\left.\frac{z p^{\prime}(z)}{p(z)}\right|_{z=z_{0}}\right)\right| \\
& =k\left|a+\frac{1}{a}\right| \geq 2 .
\end{aligned}
$$

But the results in (20) are contradictions with the assumptions in (9) and (10), respectively. Hence, the equality in (15) yields that $\mathfrak{R} e(p(z))>0$ for all $z$ in $\mathbb{U}$. Therefore, we evidently receive the inequality in (12). This completes the desired proof.

As we emphasized before, Theorem 2 includes several comprehensive results in relation with AGFT. Specially, some of its consequences containing results between certain complex (differential) equations constituted by certain operators consisting of fractional calculus and the theory of univalent functions are fairly important results. Accordingly, we want to focus on only one of them (which is Proposition 3 below) and also its two useful applications (which are Corollaries 4 and 5 below). The other possible consequences of the main result (which are here omitted) are presented to the attention of the researchers who have been working on the theory of differential equation and/or univalent function.

Proposition 3. Let $\psi(z)$ be an analytic function and satisfy any of the inequalities given by (9), and also let $\mathscr{F}(z)$ be defined by (10). If $\mathscr{F}(z)$ satisfies the following complex equation:

$$
\begin{aligned}
& {\left[\beta J_{z}^{2+\beta}[\mathscr{F}]+(\psi(z)+2 \beta-1) J_{z}^{1+\beta}[\mathscr{F}]\right] \cdot J_{z}^{\beta}[\mathscr{F}]} \\
& \quad+(1-\beta)\left[J_{z}^{1+\beta}[\mathscr{F}]\right]^{2}-\gamma \psi(z)\left[J_{z}^{\beta}[\mathscr{F}]\right]^{2}=0,
\end{aligned}
$$

then

$$
\mathfrak{R} e\left(\frac{J_{z}^{1+\beta}[\mathscr{F}]}{J_{z}^{\beta}[\mathscr{F}]}\right)>\gamma \quad(0 \leq \gamma<1 ; \beta \in \mathbf{R} ; z \in \mathbb{U}) .
$$

Proof. By taking $\alpha:=1+\beta(0 \leq \beta<1)$ in Theorem 2 and also using the following well-known identity:

$$
z \cdot\left(J_{z}^{\beta}[\mathscr{F}]\right)^{\prime}=(1-\beta) J_{z}^{1+\beta}[\mathscr{F}]+\beta J_{z}^{\beta}[\mathscr{F}],
$$

the proof of Proposition 3 can be easily proven. Its detail is here omitted.

By letting $\beta:=0$ and $\lambda:=0$ in Proposition 3 (or equivalent choosing in Theorem 2), the result (which is Corollary 4 below) relating to starlikeness of order $\gamma$ can be next obtained.

Corollary 4. Let $\psi(z)$ be an analytic function and satisfy any of the inequalities given by (9). If the function $w:=f(z) \in \mathcal{S}$ satisfies the following nonlinear complex differential equation:

$$
z\left[z w^{\prime \prime}+(1-\psi(z)) w^{\prime}\right] w-z^{2}\left[w^{\prime}\right]^{2}+\gamma \psi(z) w^{2}=0
$$

then $w \in \mathcal{S}^{*}(\gamma)$, where $0 \leq \gamma<1$ and $z \in \mathbb{U}$.

By setting $\beta:=0$ and $\lambda:=1$ in Proposition 3 (or equivalent choosing in Theorem 2), the result (which is Corollary 5 below) dealing with convexity of order $\gamma$ can be also obtained.

Corollary 5. Let $\psi(z)$ be an analytic function and satisfy any of the inequalities given by (9). If the fuction $w:=f(z) \in \mathcal{S}$ satisfies the following nonlinear complex differential equation:

$$
\begin{aligned}
& z\left[z w^{\prime \prime \prime}+(1-\psi(z)) w^{\prime \prime}\right] w^{\prime} \\
& \quad-z^{2}\left[w^{\prime \prime}\right]^{2}-(1-\gamma) \psi(z)\left[w^{\prime}\right]^{2}=0,
\end{aligned}
$$

then $w \in \mathscr{K}(\gamma)$, where $0 \leq \gamma<1$ and $z \in \mathbb{U}$.

\section{Conflict of Interests}

The authors declare that there is no conflict of interests.

\section{Acknowledgment}

The work in this investigation was supported by TÜBİTAK (The Scientific and Technological Research Council of TURKEY) with the Project no. 105T056.

\section{References}

[1] A. A. Kilbas, H. M. Srivastava, and J. J. Trujillo, Theory and Applications of Fractional Differential Equations, Elsevier Science, Amsterdam, The Netherlands, 2006.

[2] A. A. Kilbas, O. I. Marichev, and S. G. Samko, Fractional Integral and Derivatives: Theory and Applications, Gordon and Breach Science, Yverdon, Switzerland, 1993.

[3] A. A. Kilbas and J. J. Trujillo, "Differential equations of fractional order: methodsresults and problems-I," Applicable Analysis, vol. 78, pp. 153-192, 2001.

[4] V. Kiryakova, Generalized Fractional Calculus and Applications, Longman Sci. Tech., Harlow, UK, John Wiley \& Sons, New York, NY, USA, 1994. 
[5] V. Laksmikantham, S. Leela, and J. V. Devi, Theory of Fractional Dynamic Systems, Cambridge Scientific Publishers, Cambridge, U.K., 2009.

[6] R. W. Leggett and L. R. Williams, "Multiple positive fixed points of nonlinear operators on ordered Banach spaces," Indiana University Mathematics Journal, vol. 28, pp. 673-688, 1979.

[7] K. S. Miller and B. Ross, An Introduction to the Fractional Calculus and Fractional Differential Equation, John Wiley \& Sons, New York, NY, USA, 1993.

[8] K. S. Miller and B. Ross, An Introduction to the Fractional Calculus and Differential Equations, John Wiley \& Sons, New York, NY, USA, 1993.

[9] K. B. Oldham and J. Spanier, The Fractional Calculus, Academic Press, New York, NY, USA, 1974.

[10] H. M. Srivastava and S. Owa, Eds., Univalent Functions, Fractional Calculus and Their Applications, Halsted Press, John Wiley \& Sons, New York, NY, USA, 1989.

[11] H. Pollard, "The complete monotonic character of the MittagLeffler function Erf(x)," Bulletin of the American Mathematical Society, vol. 54, pp. 1115-1116, 1948.

[12] B. Ahmad and J. J. Nieto, "Existence results for a coupled system of nonlinear fractional differential equations with three-point boundary conditions," Computers and Mathematics with Applications, vol. 58, no. 9, pp. 1838-1843, 2009.

[13] R. P. Agarwal, M. Benchohra, and S. Hamani, "A survey on existence results for boundary value problems of nonlinear fractional differential equations and inclusions," Acta Applicandae Mathematicae, vol. 109, no. 3, pp. 973-1033, 2010.

[14] A. Arara, M. Benchohra, N. Hamidi, and J. J. Nieto, "Fractional order differential equations on an unbounded domain," Nonlinear Analysis: Theory, Methods \& Applications, vol. 72, no. 2, pp. 580-586, 2009.

[15] D. Araya and C. Lizama, "Almost automorphic mild solutions to fractional differential equations," Nonlinear Analysis: Theory, Methods \& Applications, vol. 69, no. 11, pp. 3692-3705, 2008.

[16] R. I. Avery and J. Henderson, "Two positive fixed points of nonlinear operators on ordered Banach spaces," Communications on Applied Nonlinear Analysis, vol. 8, pp. 27-36, 2001.

[17] M. Belmekki, J. J. Nieto, and R. Rodríguez-López, "Existence of periodic solution for a nonlinear fractional differential equation," Boundary Value Problems, vol. 2009, Article ID 324561, 2009.

[18] B. Bonilla, M. Rivero, L. Rodríguez-Germá, and J. J. Trujillo, "Fractional differential equations as alternative models to nonlinear differential equations," Applied Mathematics and Computation, vol. 187, no. 1, pp. 79-88, 2007.

[19] Y.-K. Chang and J. J. Nieto, "Some new existence results for fractional differential inclusions with boundary conditions," Mathematical and Computer Modelling, vol. 49, no. 3-4, pp. 605609, 2009.

[20] R. Dehghant and K. Ghanbari, "Triple positive solutions for boundary value problem of a nonlinear fractional differential equation," Bulletin of the Iranian Mathematical Society, vol. 33, no. 2, pp. 1-14, 2007.

[21] K. Diethelm and N. J. Ford, "Analysis of fractional differential equations," Journal of Mathematical Analysis and Applications, vol. 265, no. 2, pp. 229-248, 2002.

[22] E. R. Kaufmann and E. Mboumi, "Positive solutions of a boundary value problem for a nonlinear fractional differential equation," Electronic Journal of Qualitative Theory of Differential Equations, no. 3, pp. 1-11, 2008.
[23] V. Lakshmikantham and S. Leela, "Nagumo-type uniqueness result for fractional differential equations," Nonlinear Analysis: Theory, Methods \& Applications, vol. 71, no. 7-8, pp. 2886-2889, 2009.

[24] V. Laksmikantham and A. S. Vatsala, "Basic theory of fractional differential equations," Nonlinear Analysis: Theory, Methods \& Applications, vol. 69, pp. 2677-2682, 2008.

[25] W. R. Schneider, "Completely monotone generalized MittagLeffler functions," Expositiones Mathematicae, vol. 14, pp. 3-16, 1996.

[26] S. Zhang, "Monotone iterative method for initial value problem involving Riemann-Liouville fractional derivatives," Nonlinear Analysis: Theory, Methods \& Applications, vol. 71, no. 5-6, pp. 2087-2093, 2009.

[27] S. Z. Rida, H. M. El-Sherbiny, and A. A. M. Arafa, "On the solution of the fractional nonlinear Schrödinger equation," Physics Letters A, vol. 372, no. 5, pp. 553-558, 2008.

[28] S. Zhang, "The existence of a positive solution for a nonlinear fractional differential equation," Journal of Mathematical Analysis and Applications, vol. 252, no. 2, pp. 804-812, 2000.

[29] F. Zang, "Existence results of positive solutions to boundary value problem for fractional differential equation," Positivity, vol. 13, no. 3, pp. 583-599, 2008.

[30] O. Altintaş, H. Irmak, and H. M. Srivastava, "Fractional calculus and certain starlike functions with negative coefficients," Computers and Mathematics with Applications, vol. 30, no. 2, pp. 9$15,1995$.

[31] M.-P. Chen, H. Irmak, and H. M. Srivastava, "Some families of multivalently analytic functions with negative coefficients," Journal of Mathematical Analysis and Applications, vol. 214, no. 2, pp. 674-690, 1997.

[32] M.-P. Chen, H. Irmak, and H. M. Srivastava, "A certain subclass of analytic functions involving operators of fractional calculus," Computers and Mathematics with Applications, vol. 35, no. 5, pp. 83-91, 1998.

[33] H. Irmak and N. Tuneski, "Fractional calculus operator and certain applications in geometric function theory," Sarajevo Journal of Mathematics, vol. 6, no. 18, pp. 51-57, 2010.

[34] P. L. Duren, Grundlehren der Mathematischen Wissenchaffen, Springer, New York, NY, USA, 1983.

[35] A. W. Goodman, Univalent Functions, vol. 1-2, Polygonal Publishing House, Washington, DC, USA, 1983.

[36] M. Nunokawa, "On properties of non-caratheodory functions," Proceedings of the Japan Academy A, vol. 68, pp. 152-153, 1992. 


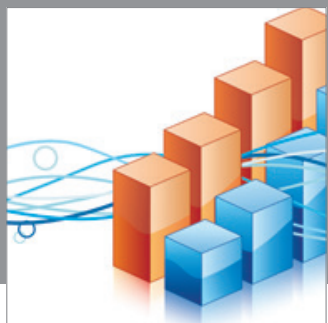

Advances in

Operations Research

mansans

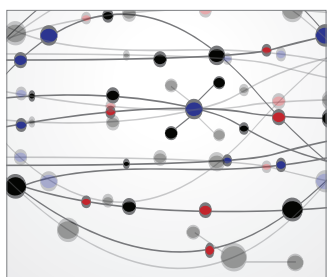

The Scientific World Journal
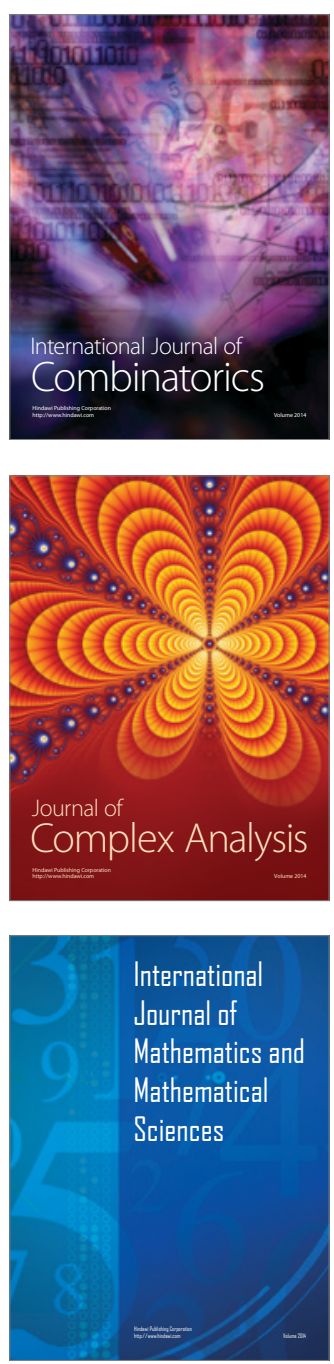
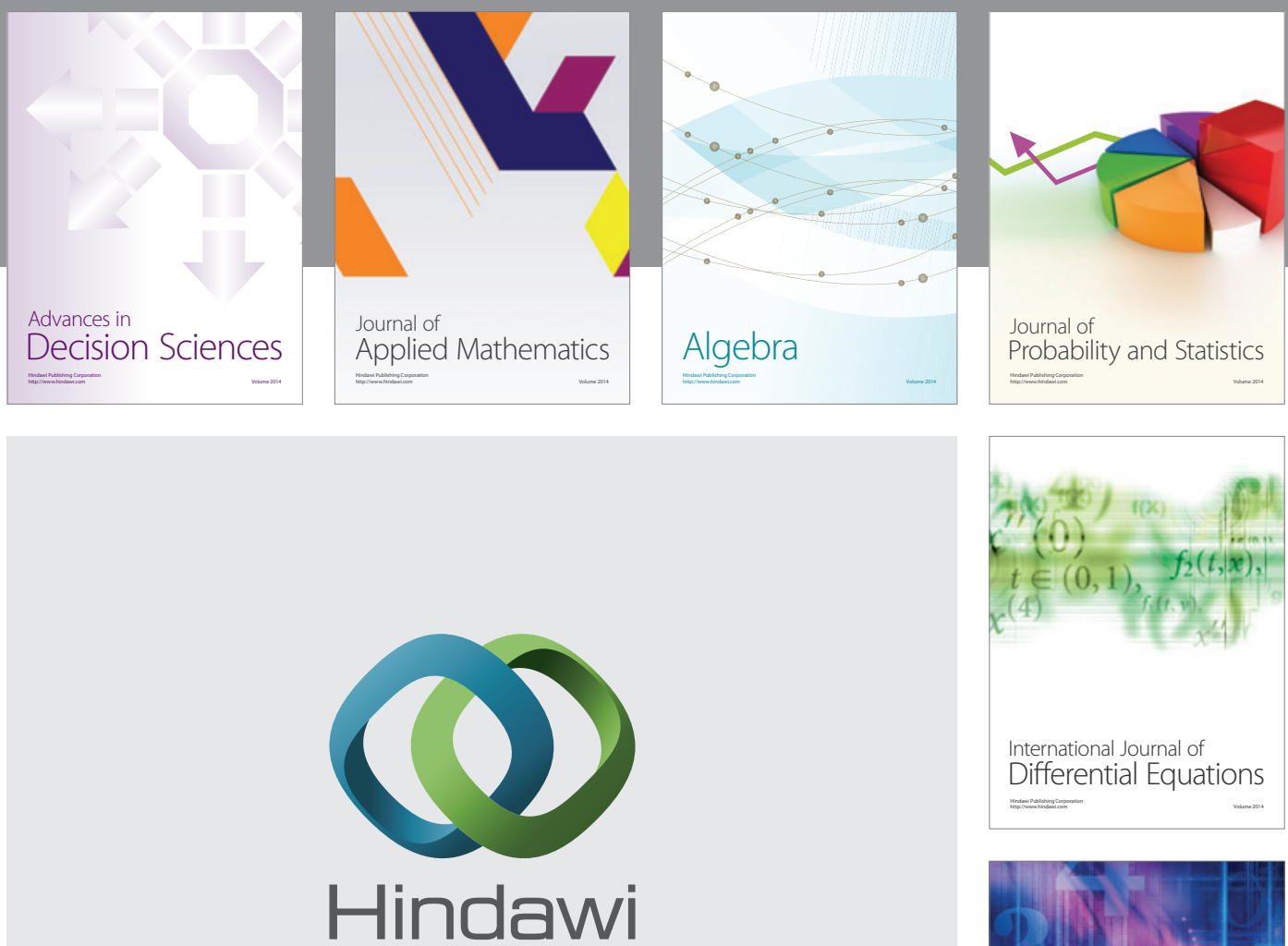

Submit your manuscripts at http://www.hindawi.com
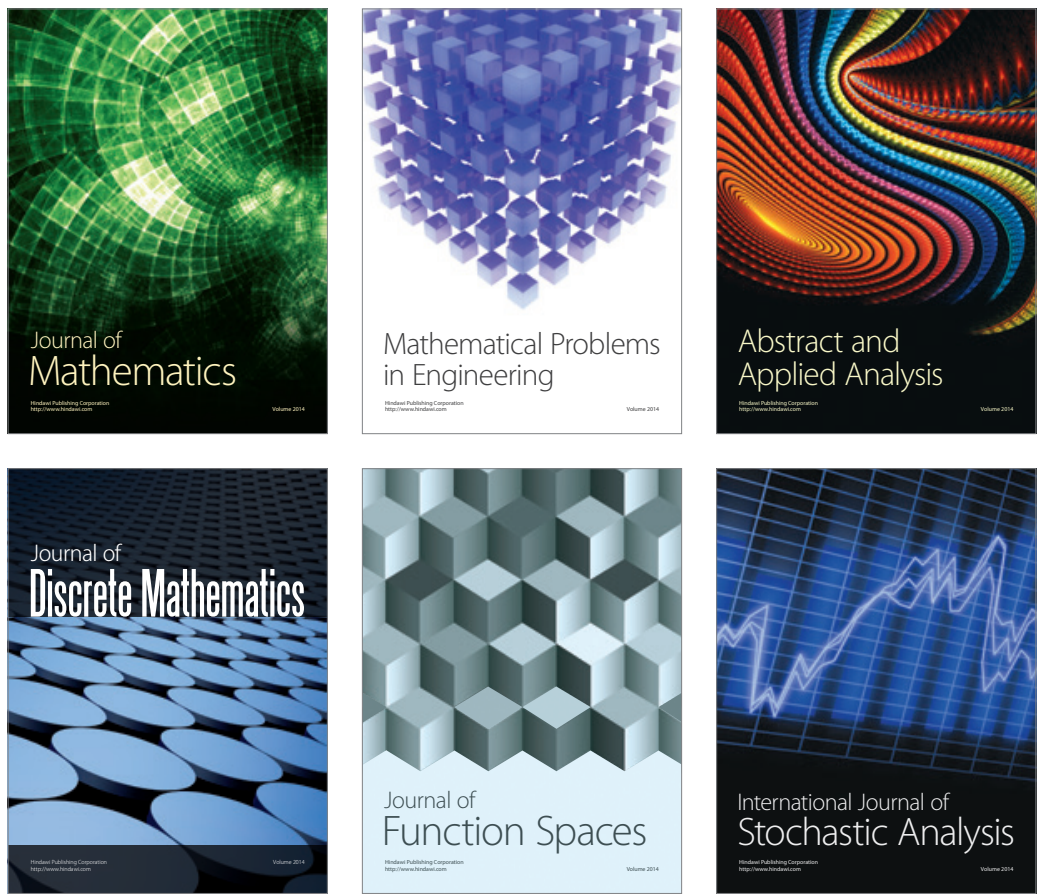

Journal of

Function Spaces

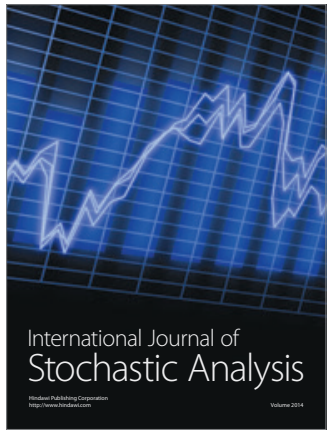

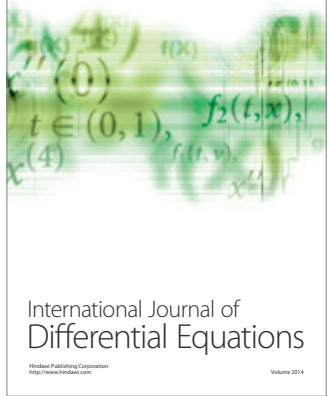
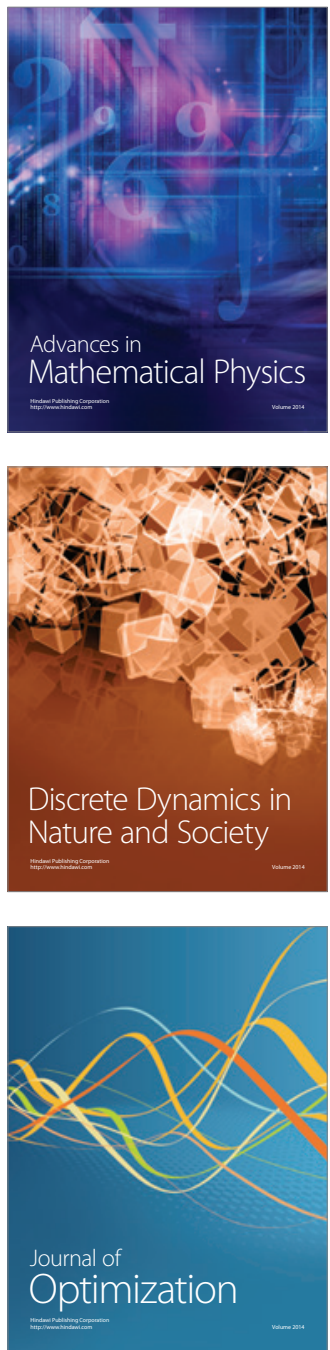